Cahiers $d u$ MONDE RUSSE

\section{Cahiers du monde russe}

Russie - Empire russe - Union soviétique et États indépendants

40/3 | 1999

Varia

\title{
A. de Lazari, ed., Idei $v$ Rossii. Idee w Rosji. Ideas in Russia. Leksykon rosyjsko-polsko-angielski
}

(Les idées en Russie. Dictionnaire russe-polonais-anglais). Varsovie, Semper, Vol. 1, 1999

Jean-Philippe Jaccard

\section{CpenEdition}

Journals

Édition électronique

URL : https://journals.openedition.org/monderusse/24

DOI : $10.4000 /$ monderusse. 24

ISSN : $1777-5388$

Éditeur

Éditions de l'EHESS

\section{Édition imprimée}

Date de publication : 1 avril 1999

Pagination : 547-550

ISBN : 2-7132-1321-5

ISSN : $1252-6576$

Référence électronique

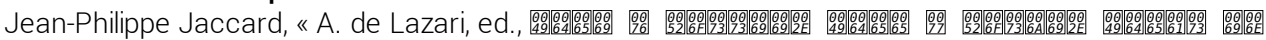

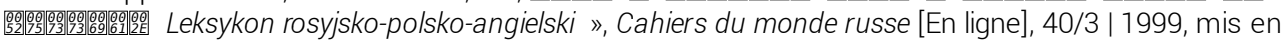
ligne le 15 janvier 2007, consulté le 02 septembre 2022. URL : http://journals.openedition.org/ monderusse/24; DOI : https://doi.org/10.4000/monderusse.24

Ce document a été généré automatiquement le 2 septembre 2022.

Tous droits réservés 


\section{A. de Lazari, ed., Idei v Rossii. Idee w Rosji. Ideas in Russia. Leksykon rosyjsko-polsko-angielski}

(Les idées en Russie. Dictionnaire russe-polonais-anglais). Varsovie, Semper, Vol. 1, 1999

Jean-Philippe Jaccard

1 Le titre en est simple, le résultat impressionnant... quant au projet, celui de « mettre un pays en idées ", il est grandiose, même si, à n'en pas douter, ce livre est appelé à susciter la méfiance et la critique, aussi bien en raison de sa conception que de sa réalisation. La Russie a en effet produit des « idées » en grand nombre, avec chaque fois des débats sans fin, débats que cet ouvrage contribuera à relancer et à enrichir.

2 De quoi s'agit-il ? Les idées en Russie se présente comme un dictionnaire encyclopédique des concepts philosophiques, théologiques, sociaux et politiques, ainsi que des penseurs qui ont façonné depuis le Moyen Age jusqu'à aujourd'hui la « culture idéologique » russe, dans le sens le plus large possible de ce mot. Le champ embrassé est extrêmement vaste, comme on le comprend dès les premières pages : après le bel hommage que constitue, indépendamment des aléas de l'alphabet, la première entrée, qui est consacrée à Sergej Averincev, on trouvera toutes sortes d'articles qui peuvent sembler au départ fort disparates, par exemple: "Akmeizm », "Antisemitizm ", "Bol’ševizm ", "Men'ševizm ", "Marrizm ", mais également «Dvorjanstvo» (Noblesse), «Dvoeverie » (Double-foi), «Zakon" (Loi), "Slavjano- fil'stvo» (Slavophilisme), "Obščina» (Communauté rurale), "Narodnost'» (Idée nationale), "Sobornost'» (Conciliarité), "Moskva - Tretij Rim " (Moscou - Troisième Rome), «Svjatost'» (Sainteté), «Domostroj» ( Ménagier»), "Jurodstvo" (Fols en Christ), ou encore "Narod» (Peuple), "Amerika», "Flagi» (Drapeaux), "Kalendar' " (Calendrier), "Geral'dika » (Héraldique), etc. Parmi les penseurs on trouvera Simeon Polockij, Konstantin Aksakov, Aleksandr Gercen, Petr Čaadaev, Nikolaj Černyševskij, Petr Lavrov, Pavel Miljukov, Nikolaj Berdjaev et bien d'autres. Ouvrage composite donc, mais à première vue seulement, car peu à peu, au-delà du tableau des idées, des notions ou des faits de culture spécifiques, se dégage une certaine 
image, qui est à la fois celle que la Russie a d'elle-même et celle qu'elle donne (ou veut donner) d'elle à l'autre, mais encore celle que cet autre se fait d'elle. Car si une douzaine d'auteurs sont russes (parmi eux: Ivan Esaulov, Andrej Delazari, Boris Egorov, Konstantin Isupov, Larisa Šilova, Ol'ga Gončarova, Sergej Gončarov), l'ouvrage est résolument polonais. On y retrouve, aux côtés de Andrzej de Lazari (professeur aux universités de Łódź et de Toruń et éditeur scientifique du projet), les noms bien connus de la russistique polonaise : Grzegorz Przebinda, Jerzy Faryno, Wassilij Szczukin, Lucjan Suchanek et bien d'autres. On est donc tout près et en-dehors à la fois. La position est certainement dangereuse (on entend déjà des voix s'offusquer: «Comment un Polonais ose-t-il ?»), mais le point d'observation est assurément exceptionnel.

3 L'ouvrage est imprimé sur quatre colonnes, dont les trois premières donnent en trois langues (russe, polonais, anglais) l'article sur l'«idée» ou le penseur traité. S'il s'agit d'un penseur, celui-ci est étudié du point de vue de sa pensée et de son impact dans l'histoire des idées exclusivement (c'est-à-dire sans données biographiques à part les dates). Quant à la quatrième colonne, elle est réservée aux données bibliographiques essentielles sur le sujet. C'est là un des points forts du livre: bien documentée, cette bibliographie est en outre intelligente, car elle ne fait pas double emploi avec ce que l'on trouve dans tous les dictionnaires et prend en compte les publications jusqu'à une période très récente, ce qui permet de faire le point sur l'état actuel de telle ou telle question.

4 Par sa forme, le livre s'adresse donc à un lectorat très large. Tout lecteur y trouvera des choses intéressantes, aussi bien le spécialiste de la Russie que l'étudiant, ou celui qui s'intéresse simplement à ce pays. Mais c'est peut-être là que l'on peut déceler une certaine faiblesse. En effet, il est important de savoir à qui on s'adresse quand on écrit des articles de ce type. Or le lecteur potentiel du dictionnaire est ici très difficile à cerner : peut-on écrire un même article pour un Russe ou un spécialiste de la Russie (qui n'a d'ailleurs pas besoin de traduction), ou pour un étudiant polonais ou américain? Une certaine vulgarisation utile pour ceux-ci ne risque-t-elle pas de tomber dans des généralités bien connues pour ceux-là? L'usage le dira, d'autant plus qu'il est encore difficile de juger véritablement du contenu, vu que seul le premier des quatre tomes est publié pour l'instant et que chaque tome regroupe des articles classés de A à Z (avec un index en trois langues à la fin de chaque volume et un index général promis à la fin du quatrième). Ce ne sera donc que lorsque l'on aura l'ensemble des quelque 600 articles prévus que l'on pourra se poser la question des choix et, par conséquent, tirer des conclusions sur la véritable ligne rédactionnelle.

Certains petits défauts et certaines grandes qualités sont toutefois d'ores et déjà perceptibles dans ce premier volume. Commençons par les premiers.

6 Ce dictionnaire, on l'a dit, est le fruit d'une ambition très grande, peut-être même un peu trop grande: c'est pourquoi il était difficile d'imaginer qu'il échappât à certaines simplifications ou à certains raccourcis, ce qui est toujours un peu dangereux quand on se place sur le terrain des idées. En outre, on observe quelques déséquilibres dans l'importance accordée à tel ou tel thème. Par exemple, l'« Ob̌̌čina » est traitée sur moins de deux colonnes (avec, il est vrai, des renvois à d'autres entrées comme "Slavjanofil'stvo ", « Narodničestvo » (Populisme) ou "Stolypin »), alors que les articles (très intéressants au demeurant) "Flagi», "Geral'dika», ou encore "Alfavit» (Alphabet), occupent chacun un espace deux à trois fois plus important. Le déséquilibre est perceptible également dans les cas où un sujet a droit à plusieurs articles (deux pour 
"Sobornost'", trois pour "Svjatotst'", par exemple). Bien sûr, il y a le désir louable d'amener le débat au coeur de l'ouvrage et d'élargir le champ de la discussion, mais vu l'absence d'un critère qui justifierait ces différences de longueur ou de traitement d'un article à l'autre, on a parfois l'impression que le hasard s'est substitué à une ligne rédactionnelle claire... Là encore, bien sûr, il faudra attendre les trois volumes suivants pour le savoir.

7 Néanmoins, la réponse à ces quelques critiques est relativement simple : si l'ambition est grande, Andrzej de Lazari, lui, est modeste, et il ne prétend aucunement offrir un ouvrage exhaustif et définitif. Il espère, essentiellement, susciter le débat autour de questions complexes qui, par définition, ne peuvent rester figées.

Cela nous amène à la qualité essentielle de ce dictionnaire : davantage qu'une véritable encyclopédie, il convient de le considérer comme le lieu d'un débat ouvert. Il fallait un certain courage pour se lancer dans une entreprise de ce genre, dans laquelle tout peut être sujet à critique et à polémique. Mais à une époque où la Russie cherche à se redéfinir, discuter de questions aussi sensibles que le fascisme russe, la question nationale ou l'antisémitisme, c'est faire oeuvre utile, d'autant plus que le regard est posé de l'extérieur, loin des affrontements idéologiques, presque toujours partisans, souvent excessifs, parfois même hystériques, du " centre ».

9 Car ce livre a bel et bien une ligne idéologique, dans le sens le moins péjoratif que ce terme peut avoir. La voix de Lazari n'est d'ailleurs pas nouvelle dans ces débats et nombreux sont ses écrits sur la question nationale en Russie ${ }^{1}$. C'est d'ailleurs lui qui signe les articles les plus sensibles, c'est-à-dire ceux qui ont trait au nationalisme russe ou à des notions voisines. Par exemple, l'article sur le droit («Pravo»), qui commence par l'affirmation qu'en Russie «il n'y a pas la conscience ancrée que l'existence et l'observation du droit soit une nécessité ", s'oriente tout entier sur la démonstration de ce postulat et sur ses conséquences: absence d'intérêt, de Radiščev à Lenin, pour les problèmes juridiques; opposition pour les Russes du «droit extérieur» occidental au «droit intérieur (moral)» russe et contraste entre l'aspiration occidentale (et égoïste) à la libération individuelle (Je) et l'esprit collectiviste dominant en Russie (Nous), etc. : "Dans la conscience russe le sujet-collectif l'emporte toujours sur l'individu-citoyen (Nous : orthodoxes, Nous : le peuple russe, ou Nous : le peuple soviétique)». Et Lazari de conclure : «Le nationalisme russe, comme tout autre d'ailleurs, relègue à l'arrière-plan les droits de l'individu-citoyen et insiste sur les droits du peuple-collectif ».

Bien d'autres articles comme celui-ci vont, à coup sûr, entraîner de vives réactions. Bien entendu, une fois de plus, il faudra attendre les volumes suivants pour se faire une idée claire du contour idéologique de l'oeuvre, mais on sait d'ores et déjà qu'une des tâches que Lazari s'est fixée est de cerner les multiples aspects du nationalisme russe, au sens le plus large de ce terme, c'est-à-dire plutôt au sens d'«idée nationale ». On peut donc s'attendre à une tempête de cris furieux : en effet un tel livre est obligatoirement idéologique, et celui-ci a la grande qualité de ne pas esquiver les questions délicates, et d'y répondre avec une louable fermeté. Les discussions qui s'ensuivront seront finalement la marque du succès de cet ouvrage destiné sans aucun doute à figurer sur les rayons de toutes les bibliothèques de russe et chez tous ceux qui s'intéressent à la Russie.

11 P.S. Au moment où nous mettons sous presse, nous apprenons que le volume 2 a paru et que l'éditeur a changé : « Ibidem » à Kurowice. 


\section{NOTES}

1.. Cf. A. de Lazari, « Poczwiennictwo ». Z badań nad historiĄ idei w Rosji, Łódź, 1988 ; id., Czy Moskwa bedzie Trzecim Rzymem ? Studia o nacjonaliźmie rosyjskim, Katowice, 1996 ; id., Nationalism in the USSR. Problems of nationalities, Amsterdam, 1989.

\section{AUTEUR}

\section{JEAN-PHILIPPE JACCARD}

Faculté des lettres, Université de Genève. 